\title{
Combining design and operational optimization of a NZEB: The Solar Decathlon China 2018 SCUTxPoliTo Prototype
}

\author{
Alessio Messina $^{1}$, Maria Ferrara ${ }^{1}$, Ciro Lisciandrello ${ }^{1}$, Enrico Fabrizio ${ }^{1}$ \\ ${ }^{1}$ TEBE Research Group, Department of Energy, Politecnico di Torino, Torino, Italy
}

\begin{abstract}
Solar Decathlon competitions are a unique opportunity to advance in research and applications in the field of NZEB design. The design of a prototype in this context can be seen as a complex optimization problem, where the objective function is the final contest score to be maximized. This paper presents the application of an original simulation-based optimization approach to support the entire process from design to construction to operation of a real NZEB prototype for Solar Decathlon China 2018. It relies on the synergic use of TRNSYS ${ }^{\circledR}$, $\mathrm{GenOpt}^{\circledR}$ and $\mathrm{Matlab}^{\circledR}$. The method resulted to be effective, leading the team to win the competition.
\end{abstract}

\section{Introduction}

In the context of the worldwide efforts to reduce energy consumptions and carbon dioxide $\left(\mathrm{CO}_{2}\right)$ emissions related to the building sector, the concept of nearly zero energy building (NZEB) has emerged as the main driver towards these objectives. It has been proved that reaching the NZEB target is technically feasible (Ferrara, 2016). However, special attention has to be paid to the transition from building design to building operation, as the designed performance does not always correspond to the actual performance after construction, therefore leading to the so-called "performance gap" (Wilde, 2014).

Among the successful experiences of design and construction of NZEBs, the Solar Decathlon competition is a unique opportunity to advance in research in the field of NZEB design. In fact, the integrated process from design to construction that is carried out for the competition purposes, the advanced required monitoring systems, the large amount of collected data and the highly interdisciplinary team working together from design to construction constitutes a fertile base for research that is hard to find in other contexts.

Since the first edition, which took place in Washington D.C. in 2002, the main purpose of Solar Decathlon is to put together teams of students with architectural and engineering backgrounds to give life to a real residential NZEB prototype. The contest is based on ten subcontests, where the teams need to achieve the highest possible score to win the competition. The total score is 1000 points, divided into 100 points for each sub-contest. Five of them investigates the project, the documentation, and the systems (Architecture, Market Appeal,
Engineering, Communication, Innovation - subjected to a jury review), while the other five focus on measured parameters, related to the comfort of occupants, the efficiency of the systems, and the energy production (Comfort Zone, Appliances, Home Life, Commuting, Energy). So, the NZEB is judged on a complex system of parameters characterizing its performance and its liveability.

In this context, the design of the NZEB prototype can be seen as a complex optimization problem, where the objective function is the final contest score to be maximized. There are a lot of involved parameters, each one may impact at the same time energy consumption and production, comfort, operation time.

A traditional parametric design approach may lead to spend and waste a lot of time on useless design variables (Ferrara, 2014). If other important design variables are not considered, it could lead even to a wrong solution. It is recognized that simulation-based methods are powerful tools for effectively solving this kind of complex problems while saving time (Nguyen, 2014). In fact, they help reduce the high computational cost needed to check a great number of design alternatives while ensuring a considerable accuracy in finding the optimal design solution.

In building science, such simulation-based optimization methods have been applied to many different problems, like energy consumption reduction ( $\mathrm{Li}, 2017)$, efficiency of systems (Wu, 2018), indoor comfort, and mostly on the costs with a life cycle perspective (Ferrara, 2018). Moreover, some studies are performed at the design stage, while others focus on the operation stage. It is hard to find examples of real NZEB buildings that are optimized using a simulation-based optimization method that considers parameters related to design and operation at the same time. It is even harder to find studies when the method is tested and validated in practice.

In 2018, the Solar Decathlon China was won by a team composed of students from Politecnico di Torino and the South China University of Technology (SCUT-POLITO team), thanks to the application of simulation-based optimization methods to maximise the contest score.

\section{Objectives of the work}

Based on the Solar Decathlon context, this work aims at combining design and operation parameters to optimize a real residential NZEB prototype and to validate results 
throughout the entire process from design to construction to operation.

To reach this main goal, the proposed approach is based on different steps:

- Creation of a BEM (Building Energy Model) for the NZEB prototype;

- Implementation of the Contest Schedules;

- Definition of the objective function based on the Solar Decathlon competition rules;

- Set up a simulation-based optimization framework able to optimize the performance of a NZEB building (coupling of TRNSYS, MATLAB, GenOpt);

- Design variation on the base of the results.

\section{The LONGPLAN prototype}

The NZEB prototype, whose name is LONG PLAN (Fig.1), is based on the concept of the narrow house to face the problem of high-density city urbanization and land consumption. It has two floors, the net floor area is about $143 \mathrm{~m}^{2}$, it has a modular steel structure with 12 prefabricated modules.

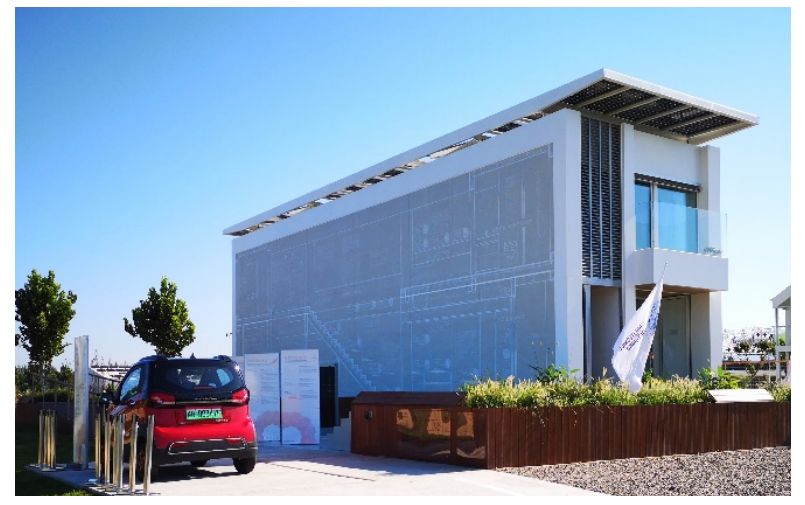

Figure 1: Picture of the LONG PLAN house
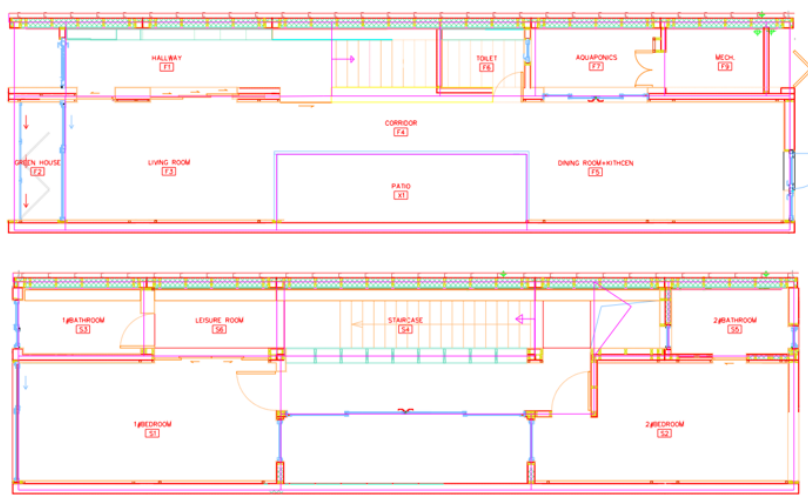

Figure 2: Plans of the LONG PLAN house

As it can be seen from the plans in Figure 2, the building is a two-storey house that can be divided into three main volumes, also called "belts":

- Implemented Wall: The west-side external wall containing all the distribution pipes for hot water, coolant, DHW and all the electrical and electronic connection, designed to limit the use of space and to make the maintenance easier;

- Service Belt: A narrow "slice" of the house, composed of all the services and systems. It includes the stairs, the 3 bathrooms, the mechanical room, the aquaponic system and the kitchen appliances;

- Living Belt: This section is composed by the 4 conditioned zones: living room, kitchen and the two bedrooms. There is also one corridor for each floor and a central patio having an automated roof windows, designed to improve passive strategies such as the chimney effect.

Transparent envelope $\left(\mathrm{U}_{\mathrm{w}}\right.$ ranging from $0.8 \mathrm{~W} / \mathrm{m}^{2} \mathrm{~K}$ to $1.2 \mathrm{~W} / \mathrm{m}^{2} \mathrm{~K}$ ) is only on north and south facades (shortest sides), plus two skylights near the staircase, because the longest walls are supposed to be adjacent to the other houses. The opaque envelope is composed of OSB (Oriented Strain Board) panels, VIP (Vacuum Insulated Panels), phenolic insulation, water barrier, vapor barrier (roof and walls: $\mathrm{U}=0.095 \mathrm{~W} / \mathrm{m}^{2} \mathrm{~K}$; ground slab: $\mathrm{U}=0.129$ $\mathrm{W} / \mathrm{m}^{2} \mathrm{~K}$ ). The east and west facades are heavily insulated to simulate the adjacent house and avoid extra-gains, and a ventilated façade is added to limit solar gains.

In the city of Dezhou, Shandong, China, thermal loads were estimated to be $16 \mathrm{~kW}$ for cooling and $9 \mathrm{~kW}$ for heating. To cover these loads, the HVAC system (Fig. 3) of the prototype was developed focusing on the modularity and feasibility of the project. All the technologies applied for the systems are market-available. The cooling system is composed of a Variable Refrigerant Volume (VRV-Daikin $\left.{ }^{\circledR}\right)$ Heat Pump connected with four internal units with enhanced dehumidification capability. The heating system is composed of a 4-loop capillary heating system that is fed by the same external heat pump through a high efficiency heat exchanger. The four loops are independent, the mats are pre-casted inside the concrete of the floor in the main conditioned rooms.

There is a recycling system for the grey water and condensate from the HVAC system, which saves around half of a typical water consumption, and uses the purified water to different purposes: plants feeding, toilet flush, sprinkler system, rain garden.

The ventilation system was designed to reduce the $\mathrm{CO}_{2}$ and $\mathrm{PM}_{2.5}$ (fine particulate matter) concentration in the indoor air. An Energy Recovery Ventilator was designed to provide a fresh air flow of $350 \mathrm{~m}^{3} / \mathrm{h}$. The outdoor air is firstly filtered in a coarse filter and then in a finer one, obtaining a filtering efficiency to the PM2.5 $>99 \%$. The air then passes through a counter flow heat exchanger in which it exchanges sensible energy with the exhaust air to reduce the conditioning load on the inside. The primary air is supplied directly to the living belt, in the 4 conditioned rooms, while the extraction is located in the corridor of the first floor and next to the top of the aquaponic system on the second floor (this position was chosen to highly reduce the amount of humidity near the green-wall). The flow path is ensured by the normal air leakage of the internal doors without increasing the pressure drops considerably. 
On the roof, $11 \mathrm{~kW}_{\mathrm{p}}$ of high-efficiency PV panels are installed on a steel structure. There are also $6 \mathrm{~m}^{2}$ of solar thermal panels to produce domestic hot water, combined to an electrical resistance to increase availability and temperature control.

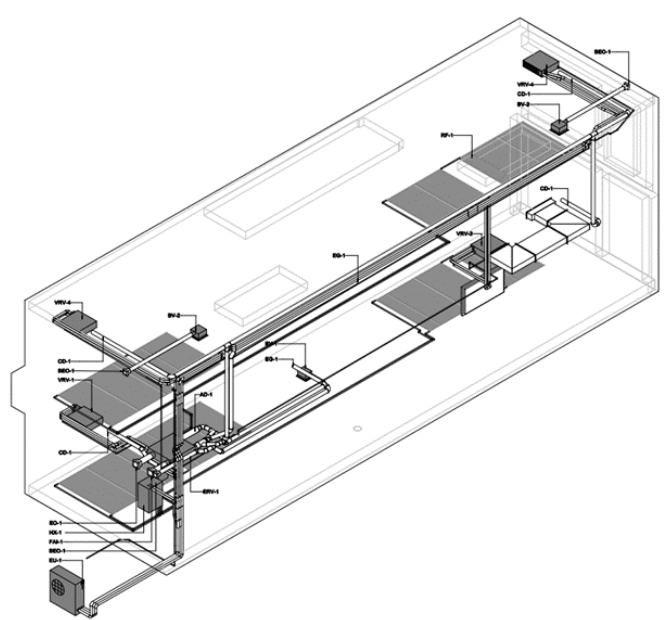

Figure 3: 3-D Schematic of the prototype HVAC system

\section{Methods}

As mentioned, the proposed automated optimization framework is based on the coupling between a simulation program and an optimization engine; the problem, therefore, is solved through iterative methods guided by optimization algorithms.

As shown in Figure 4, this study relies on the use of TRNSYS, supported by CONTAM, to set up the building model and perform detailed energy simulations. MATLAB is used to manage simulation outputs and calculate the cost function, while GenOpt is used to set up optimization variables and drive the optimization process.

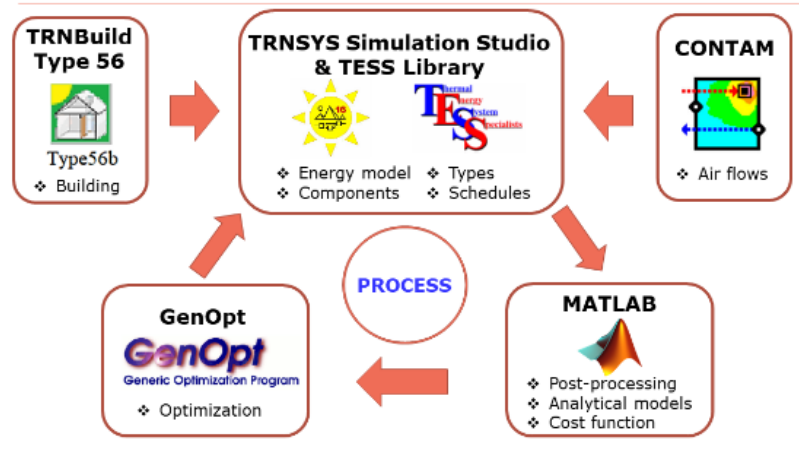

Figure 4: Optimization framework and used tools

\section{Optimization objective}

As a first step to set up the optimization framework, the optimization objective has to be translated into a cost function that is computed by the involved tools. As mentioned, the objective is to maximize the contest score. For this purpose, only the sub-contests related to measured variables were considered as the others were judged according to non-objective criteria. The set of objective criteria related to zone variables that can be monitored, simulated and therefore optimized to maximize their contribution to the final score are listed below.

Temperature -40 points

- $\quad$ Full points in the range $22-25^{\circ} \mathrm{C}$

- $\quad$ Linearly reduced point between $22-19{ }^{\circ} \mathrm{C}$ and 25 $28^{\circ} \mathrm{C}$

- Zero points in all other cases.

- Relative Humidity - 20 points;

- Full points in the range 0-60\%

- $\quad$ Linearly reduced point between $60-70 \%$

- Zero points in all other cases.

- $\mathbf{C O}_{2}-20$ points;

- $\quad$ Full points in the range $0-1000 \mathrm{ppm}$

- $\quad$ Linearly reduced point between 1000-2000 ppm

- Zero points in all other cases.

- $\quad$ PM2.5 - 20 points;

- Full points in the range $0-35 \mu \mathrm{g} / \mathrm{m}^{3}$

- Linearly reduced point between $35-75 \mu \mathrm{g} / \mathrm{m}^{3}$

- Zero points in all other cases.

- Energy balance - 80 points;

Based of the difference between PV production and energy consumption during the contest.

- $\quad$ Full points for positive production $(>0 \mathrm{kWh})$

- $\quad$ Linearly reduced point between 0 and $-50 \mathrm{kWh}$

- Zero points in all other cases.

The above-listed criteria are related to a maximum of 180 points out of 1000 . Such points may be decisive for final ranking, considering that usually the Solar Decathlon teams compete for the first positions with tiny differences of points.

The cost function was implemented in MATLAB and linked to the input variables simulated in the TRNSYS model, considering the complex schedules of the contest. As mentioned, the contest period lasted for two weeks, each day having different tasks with different schedules (tasks include domestic energy-consuming activities like doing the laundry, preparing meals, or inviting friends for a party). Points are calculated, day by day, assigning a part of the total score, using the mean value of the measured parameters among specified intervals of time. The energy balance and the related points are calculated at the end of the period.

\section{The energy model}

As mentioned, the building energy model to simulate the building performance in meeting the contest objectives was created in TRNSYS. Its structure in the Simulation Studio interface is reported in Figure 5. 


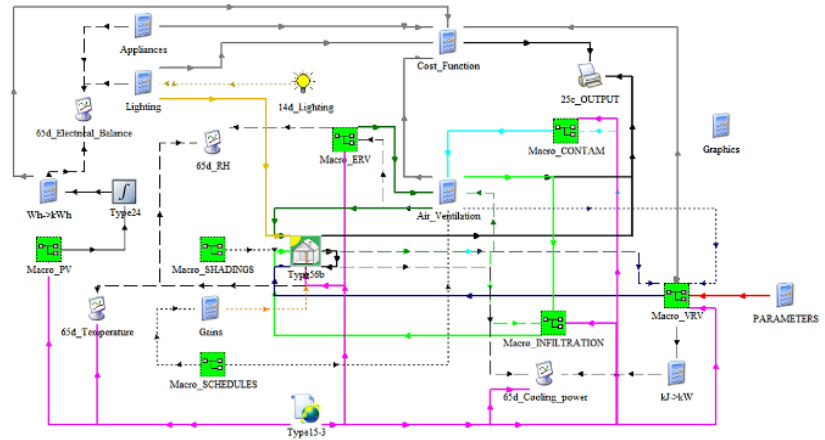

Figure 5: TRNSYS model in Simulation Studio.

The core Type for the simulation is the Type-56; it allows a detailed description of the envelope and of the different zones, simulated through a nodal configuration. The zoning considered 16 zones, of which 4 (the ones in bold in Table 1) are directly conditioned. This allows direct control of the conditioned spaces even if they are part of a bigger open space (Raftery et al., 2011): having smaller thermal zones implies a higher control on the ventilation flow path and higher precision on the temperature and humidity values in that particular part of the prototype. These characteristics are fundamental to maximize the model accuracy and reliability.

Table 1: Thermal zones in the model (ref Fig.2)

\begin{tabular}{|c|c|c|}
\hline Code & Room/Zone & Vol $\left[\mathbf{m}^{\mathbf{3}}\right]$ \\
\hline F1 & Hallway & 20.7 \\
\hline F2 & Greenhouse & 9.6 \\
\hline F3 & Living Room & $\mathbf{3 9 . 7}$ \\
\hline F4 & Corridor & 20.3 \\
\hline F5 & D.R.+Kitchen & $\mathbf{4 9 . 4}$ \\
\hline F6 & Bathroom 1 & 6.9 \\
\hline F7 & Aquaponics & 13.4 \\
\hline F8 & Mechanical Room & 10.6 \\
\hline S1 & Bedroom 1 & $\mathbf{4 9 . 3}$ \\
\hline S2 & Bedroom 2 & $\mathbf{4 4 . 3}$ \\
\hline S3 & Bathroom 2 & 12.0 \\
\hline S4 & Staircase & 81.1 \\
\hline S5 & Bathroom 3 & 12.0 \\
\hline S6 & Leisure Room & 14.6 \\
\hline X1 & Patio & 61.6 \\
\hline X2 & Cabinet & 3.0 \\
\hline
\end{tabular}

The building was then connected to different macros, each including several types simulating a system component or loop, like PV panel for the production, the ERV, the shadings, the infiltrations in the monitored zones, the contest schedules. The simulation components for VRV system were created ad hoc, based on the partial load datasheets provided by the manufacturer and on some user-defined equations calculating the actual power delivered and the energy consumption. A sub-routine from the CONTAM software was used to calculate and implement the airflows between the zones of the house. The radiant heating floor was not simulated because the contest is supposed to take place in summer, when there are no heating loads. The domestic hot water loop was also excluded from simulation, because it is not optimized in the study. Moreover, the consumption of the other tasks and appliances were calculated in the model based on the contest rules. The pollutants levels $\left(\mathrm{CO}_{2}\right.$ and PM2.5) were calculated through a user-defined analytical approach, using a build-up/decay model, checked by some measurement on the field during the test phases.

The adopted simulation time-step is 1 minute, for a more accurate evaluation of the system operation. The simulation was run in the range 5112-5472 hours of the year, corresponding to the contest planned period. The weather file selected for the simulation was the TMY file referring to Raoyan, a city close to the contest location. Before running the optimization, the described building model was calibrated (by means of optimization-based calibration carried out in a parallel study on this project) using the measured data collected on-site during the construction phase, including the location climate scenari (Ferrara, 2017a).

\section{Optimization variables}

Once the optimization objective and the energy model were defined, the design variables impacting the created cost function were identified. Twenty-four variables were selected, some related to the building envelope and others related to the system design and operation, as reported in Table 2.

Table 2: Description of selected optimization variables.

\begin{tabular}{|c|c|}
\hline Parameters & Name \\
\hline Setpoint Temperature & T_VRV \\
\hline ERV turn on time day 1 & ERV_on1 \\
\hline ERV turn on time day 2 & ERV_on2 \\
\hline ERV turn off time day 1 & ERV_off1 \\
\hline ERV turn off time day 2 & ERV_off2 \\
\hline Array Slope & PV_angle \\
\hline Shading North & Sh_N \\
\hline Shading South & Sh_S \\
\hline Shading Horizontal & Sh_H \\
\hline Shading Time ON North & Sh_N_on \\
\hline Shading Time ON South & Sh_S_on \\
\hline Shading Time ON Horizontal & Sh_H_on \\
\hline Shading Time OFF North & Sh_N_off \\
\hline Shading Time OFF South & Sh_S_off \\
\hline Shading Time OFF Horizontal & Sh_H_off \\
\hline OSB thickness, North wall & OSB_N \\
\hline OSB thickness, South wall & OSB_S \\
\hline OSB thickness, Roof & OSB_Roof \\
\hline OSB thickness, Other surfaces & OSB \\
\hline Phenolic Insulation thickness, North & INS_N \\
\hline Phenolic Insulation thickness, South & INS_S \\
\hline Phenolic Insulation thickness, Roof & INS_H \\
\hline Phenolic Insulation thickness, Other & INS \\
\hline Phenolic Insulation thickness,Int. Walls & INS_int \\
\hline
\end{tabular}

The set of optimization variables represents the set of design variables that could be controlled by the simulation model and could be checked and optimized during the 
construction and the pre-contest test phases. Their selection was performed according to real feasibility criteria, but it has to be noted that other measures related to other design variables could potentially lead to better performance in terms of energy saving and improved comfort.

The selected design-related variables refer to the tilt angle of the PV panel and the thickness of the massive and light layers of envelope (OSB and phenolic insulation). Some of them are set for different orientations, to evaluate their influence on the performance and thus on the contest score. The operation-related variables refer to the cooling setpoint, the ERV operation schedules, the shading fraction and the related time schedules.

The range of variation and the step length of the selected set of optimization variables is reported in Table 3. They were set as discrete variables in order to reflect their variation possibilities in the reality. This was done according to market availability criteria for design-related variables (e.g. the available OSB panles are $12 \mathrm{~mm}$ thick), while operation-related variables were set according to feasibility and easiness-of-use criteria (e.g. a fraction of 0.62 for the shading devices is not smart because not practically useful).

This lead to define a search space composed of $3.85 \bullet 10^{16}$ possible combinations of design-operation options.

Table3: Settings of optimization variables.

\begin{tabular}{|c|c|c|c|c|}
\hline Name & Unit & Min & Max & Step length \\
\hline T_VRV & ${ }^{\circ} \mathrm{C}$ & 20 & 25 & 1 \\
\hline ERV_on1 & hour & 16 & 18 & 0.5 \\
\hline ERV_on2 & hour & 19.5 & 21.5 & 0.5 \\
\hline ERV_off1 & hour & 19.5 & 21.5 & 0.5 \\
\hline ERV_off2 & hour & 22 & 24 & 0.5 \\
\hline PV_angle & ${ }^{\circ}$ & 0 & 25 & 5 \\
\hline Sh_N & Fraction & 0 & 1 & 0.5 \\
\hline Sh_S & Fraction & 0 & 1 & 0.5 \\
\hline Sh_H & Fraction & 0 & 1 & 0.5 \\
\hline Sh_N_on & hour & 7 & 12 & 1 \\
\hline Sh_S_on & hour & 7 & 12 & 1 \\
\hline Sh_H_on & hour & 7 & 12 & 1 \\
\hline Sh_N_off & hour & 17 & 22 & 1 \\
\hline Sh_S_off & hour & 17 & 22 & 1 \\
\hline Sh_H_off & hour & 17 & 22 & 1 \\
\hline OSB_N & $\mathrm{mm}$ & 0.012 & 0.036 & 0.012 \\
\hline OSB_S & $\mathrm{mm}$ & 0.012 & 0.036 & 0.012 \\
\hline OSB_Roof & $\mathrm{mm}$ & 0.012 & 0.036 & 0.012 \\
\hline OSB & $\mathrm{mm}$ & 0.012 & 0.036 & 0.012 \\
\hline INS_N & $\mathrm{mm}$ & 0.10 & 0.24 & 0.02 \\
\hline INS_S & $\mathrm{mm}$ & 0.10 & 0.24 & 0.02 \\
\hline INS_H & $\mathrm{mm}$ & 0.10 & 0.24 & 0.02 \\
\hline INS & $\mathrm{mm}$ & 0.10 & 0.24 & 0.02 \\
\hline INS_int & $\mathrm{mm}$ & 0.10 & 0.24 & 0.02 \\
\hline
\end{tabular}

\section{Optimization settings and runs}

As described, the optimization problem in this study is characterized by discrete variables. A meta-heuristic method was selected, belonging to the stochastic population-based family algorithms: The Particle Swarm Optimization (PSO) (Kennedy, 1995). This was selected because it does not depend on the nature of the objective function, it limits the risk of getting stuck in local optima and it leads to a great number of cost function evaluations, for a deep exploration of the search space (Machairas, 2014).

In this study, the binary PSO implementation of the generic optimization program GenOpt ${ }^{\circledR}$ (Wetter (2011)) was used, because of its ability in dealing with discrete variables.

Since the algorithm is already coded in the GenOpt scripts, it only needed to be properly set and coupled to the simulation software to the perform the optimization. The GenOpt configuration file, containing indication about the simulation program to call, was created, as well as the command file, where the optimization variables were defined and the and algorithm settings are reported.

The coupling between TRNSYS and GenOpt was finalized by editing the TRNSYS input files. The editing entails creating two template files where GenOpt is able to write different values of optimization parameters driven by the algorithm, thus creating different simulation input files at each iteration.

A MATLAB script was also created, which is called at each iteration to evaluate the cost function based on values provided by a TRNSYS output file and to report to GenOpt the objective function value. The script handles the simulation outputs in relation to the points gained for the contest purpose.

The time required for one iteration (corresponding to one simulation run) is around two minutes, in a computer equipped with a processor Intel Core I7-6700HQ (2.6 $\mathrm{GHz}$ ) and $8 \mathrm{~GB}$ RAM. The time for the optimization, obviously, grows as the number of generations and particles grows. Therefore, in order to limit the computation time, it is very important to determine how many iterations are required to reach the optimum, that is the maximization of the cost function, and how much it varies according to the number of iterations and the other algorithm settings.

Therefore, several optimization runs were performed to check the efficiency of the algorithm and the effectiveness of the tool and adjust their settings. In particular, according to the approach followed by Ferrara (2017b), different numbers of iterations were tested, varying the number of particles, the number of generations, and also different combinations of the PSO algorithm social and cognitive accelerations. It has to be noted that, because the global optimum is known (it corresponds to the 180 points for the contest score), this constitutes also a test for the ability of PSO algorithm in dealing with building optimization problems . 


\section{Results and Discussion}

The diagrams in Figures 6 and 7 reports the obtained contest points (out of 180) in the different optimization runs. In particular, Figure 6 reports the starting point (initial design and operation settings) and the percentage improvement of the score after optimization runs, which ranges between $7 \%$ and $9 \%$.

The starting score with the project data is 162.42789 . Each optimization shows an improvement of the solution, the maximum is around the value of 176.5 points. The highest score achieved is equal to 176.93825 . Because a lot of combinations of design variables leading to better scores were found, a higher number of particles could be beneficial for a better exploration of the space of solutions.

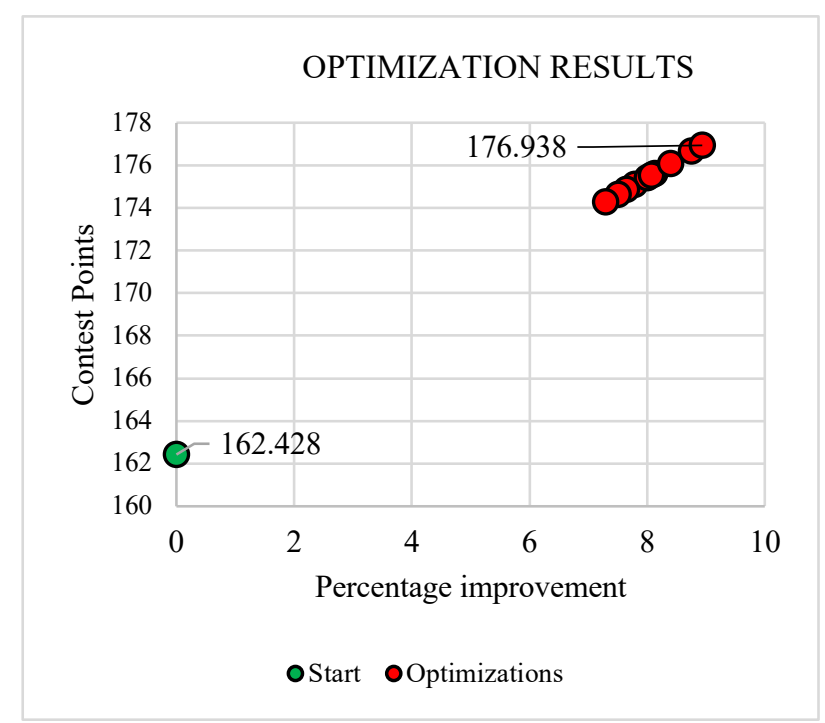

Figure 6: Contest score reached after optimization.

Therefore, the last optimization were performed with a growing number of iterations, increasing the number of particles up to 60 , and the number of generations up to 30 , allowing 1920 iterations. As shown in Figure 7, the last generations are catching more effective scores, near the maximum of 180 .

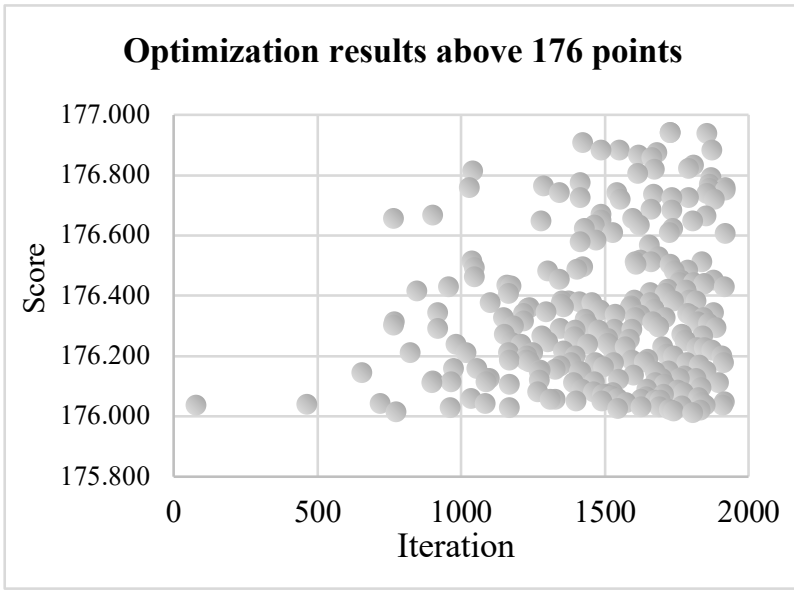

Figure 7: Iterations above 176 points.
The resulting optimal set of values to be assigned to the optimization variables are reported in Table 4 . They were defined based on the most frequent values obtained in the neighborhood of the optimum (the set of solutions obtaining score above 176 points).

Table 4: Initial and optimal values of optimization variables.

\begin{tabular}{|c|c|c|c|}
\hline Name & Unit & Initial value & Optimal value \\
\hline T_VRV & ${ }^{\circ} \mathrm{C}$ & 24 & 21 \\
\hline ERV_on1 & hour & $17: 00$ & $16: 30$ \\
\hline ERV_on2 & hour & $20: 30$ & $19: 30$ \\
\hline ERV_off1 & hour & $20: 30$ & $19: 30$ \\
\hline ERV_off2 & hour & 23:00 & $22: 00$ \\
\hline PV_angle & $\circ$ & 2 & 25 \\
\hline Sh_N & Fraction & 0 & 0 \\
\hline Sh_S & Fraction & 0 & 0.5 \\
\hline Sh_H & Fraction & 0 & 0.5 \\
\hline Sh_N_on & hour & 11:00 & $12: 00$ \\
\hline Sh_S_on & hour & 11:00 & $12: 00$ \\
\hline Sh_H_on & hour & $11: 00$ & $7: 00$ \\
\hline Sh_N_off & hour & 19:00 & $20: 00$ \\
\hline Sh_S_off & hour & 19:00 & 19:00 \\
\hline Sh_H_off & hour & 19:00 & $22: 00$ \\
\hline OSB_N & $\mathrm{mm}$ & 0.012 & 0.036 \\
\hline OSB_S & $\mathrm{mm}$ & 0.012 & 0.036 \\
\hline OSB_Roof & $\mathrm{mm}$ & 0.012 & 0.036 \\
\hline OSB & $\mathrm{mm}$ & 0.012 & 0.024 \\
\hline INS_N & $\mathrm{mm}$ & 0.20 & 0.12 \\
\hline INS_S & $\mathrm{mm}$ & 0.20 & 0.12 \\
\hline INS_H & $\mathrm{mm}$ & 0.20 & 0.14 \\
\hline INS & $\mathrm{mm}$ & 0.20 & 0.10 \\
\hline INS_int & $\mathrm{mm}$ & 0.20 & 0.18 \\
\hline
\end{tabular}

As shown in Table 4, the optimization led to modify almost all the involved design and operation variables. Such modifications can be summarized and discussed as follows:

- In order to ensure high air quality score, ERV should be turned on earlier than expected. However, to limit energy consumptions while maintanining high scores in other categories, the operation time range should be reduced by anticipating the turning off hour;

- The temperature setpoint of the VRV should be set to $21^{\circ} \mathrm{C}$, because the solar radiation and the heat stored during the day contribute to the fast rise of internal temperature;

- PV panels' tilt angle should be higher to increase the theoretical production of the given PV area. However, tilted panels requires a certain space between them to avoid mutual shadings, which could reduce the available roof area;

- The shadings on the north façade are not important, while the others (south and horizontal), must be used 
at half of their capability for a larger time range than expected;

- A higher mass should be put in the opaque envelope, as expressed through higher OSB thickness in all related variables, while insulation should be reduced.

It has to be noted that, despite the initial purposes, there was no time to implement the resulting optimal design of the opaque envelope, due to delays in the construction phase. However, this is not so problematic in the north and south façades, because of the little areas of the opaque walls and the limited impact of such measures in the final results. This becomes important for controlling the solar radiation on the opaque roof, which is connected to the optimization outcomes related to shadings. With this in mind, the resulting optimal strategies related to shadings were carefully implemented, with the model providing an objective feedback for the last-minute decisions during the contest.

Another critical aspect, that is worth to be discussed, is related to the weather data. The typical meteorological year that was used for initial simulations at the design phase resulted to be distant from the real weather conditions during the contest season. In fact, the real weather was hotter, more humid, and the sky was more covered (2 raining days) than simulated and therefore the power needed for the cooling was higher, with higher consumption too, and the electricity production less than expected.

The monitored weather data implemented in the final simulations with the calibrated model, as well as the safety range considered for the sizing and the performance assessment of the cooling system, resulted to be good strategies to keep the model close to the reality, thus confirming the strength of the optimization.

For the next construction of the house, the entire set of parameters values can be updated to improve the envelope behavior, achieving the top-performing prototype.

\section{Conclusion}

This work presents an effective transition from theoretical simulation to reality and back. The implemented simulation-based optimization methodology resulted to be effective in supporting the design, construction and management of a prototype house for the Solar Decathlon China 2018 competition.

The achieved time saving with respect to the manual comparison of different combination of variables is important, considering that the number of analysed design and operation alternatives is huge.

Most of the resulting optimal design parameters, were implemented in the construction of the house, while others were used to drive the prototype monitoring and management. In this way, it was possible to have the best overall analysis, both for the design and management phases, and win the competition.

The optimization work led to improve the contest score by more than 15 points with respect to the estimated score pre-optimization. This adds even more value to the work itself, in a context where a single point can change the outcome of the competition and the competition was won with a deviation less than 12 points with respect to the second team.

As a general conclusion, it has emerged that the accuracy of the model has a great influence on the results and therefore on the effectiveness of the approach. In particular, the parallel model calibration is fundamental to reach high performance levels. Also, a tailored sensitivity analysis can help refine the variables mesh of variation and prioritize the correct implementation of the resulting optimal strategies if external constraints (e.g. time, budget, ..) occur and will be investigated in future work.

The presented method can be applied to other prototypes, to building fine-testing, to support commissioning. It can be further applied for other competitions or for supporting the transition to market of the Solar Decathlon prototypes.

\section{Acknowledgement}

This work has been performed as part of the activities of the team SCUT-POLITO (South China University of Technology, Guangzhou, China - Politecnico di Torino, Turin, Italy), winner of the Solar Decathlon China 2018 contest ( http://www.sdchina2017-scutpolito.com).

The contribution of all the students composing the team from China and Italy is acknowledged, supervised by the followings

SCUT professors: Sun Yimin, Wang Jing, Xiao Yiqiang, Xu Haohao, Zhang Yufeng, Zhong Guanqiu.

PoliTo professors: Mauro Berta (Scientific coordinator, DAD), Michele Bonino (DAD), Orio De Paoli (DAD), Enrico Fabrizio (DENERG), Marco Filippi (DENERG), Francesca Frassoldati (DAD), Matteo Robiglio (DAD), Valentina Serra (DENERG), Edoardo Bruno (DAD).

LINKS-SiTI research group: Romano Borchiellini (DENERG), Sergio Olivero, Paolo Lazzeroni, Federico Stirano.

\section{References}

Delgarm, N., Sajadi, B., Kowsary, F., Delgarm, S. (2016) Multi-objective optimization of the building energy performance: A simulation-based approach by means of particle swarm optimization. Applied Energy 170, 293-303.

Ferrara, M., Fabrizio, E., Virgone, J., Kuznik, F., Filippi, M. (2014) Modelling Zero Energy buildings: parametric studies for the technical optimization. Energy Procedia 62, 200-209.

Ferrara, M., Fabrizio, E., Virgone, J., Filippi, M. (2016) Energy systems in cost-optimized design of nearly zero-energy buildings. Automation in construction 70, 109-127. 
Ferrara, M, Fabrizio, E. (2017a) Cost optimal nZEBs in future climate scenarios. Energy Procedia 122, 877882.

Ferrara, M, Dabbene, F., Fabrizio, E. (2017b) Optimization Algorithms Supporting The CostOptimal Analysis: The Behavior of PSO. Proceedings of BS 2107, San Francisco, USA.

Ferrara, M, Sirombo, E., Fabrizio, E. (2018). Energyoptimized versus cost-optimized design of highperforming dwellings: The case of multifamily buildings. Science and Technology for the built environment 24(5), 513-528.

Kennedy, J and Eberhart, R.C. (1995) Particle swarm optimization. In IEEE International Conference on Neural Networks, vol. IV, 1942-1948.

Kennedy, J. and Eberhart, R.C. (1997). A discrete binary version of the particle swarm algorithm. IEEE Proc. of Systems, Man, and Cybernetics 5, 4104-4108.

Li, K. Pan, L., Xue, W., Jiang, H., Mao, H. (2017) MultiObjective Optimization for Energy Performance Improvement ofed Residential Buildings: A Comparative Study. Energies 10(2), 245.
Machairas, M. Tsangrassoulis, A., Axarli, K. (2014) Algorithms for optimization of building design: A review, Renewable and Sustainable Energy Reviews 31, 101-112.

Nguyen, A-T. (2014) A review on simulation-based optimization methods applied to building performance analysis, Applied Energy 113, 10431058.

Raftery, P., Keane, M., O’Donnell, J. (2011) Calibrating whole building energy models: An evidence-based methodology. Energy and Buildings 43, 2356-2364.

Wetter, M. (2011). GenOpt Generic Optimization Program User Manual, v3.1.0.

Wilde, P. (2014) The gap between predicted and measured energy performance of buildings: A framework for investigation. Automation in Construction 41, 40-49.

Wu, W., Guo, J., Li, J., Hou, H., Meng, Q., Wang, W. (2018) A Multi-objective Optimization Design Method in Zero Energy Building Study: A Case Study Concerning Small Mass Buildings in Cold District of China. Energy and Buildings 158, 16131624 